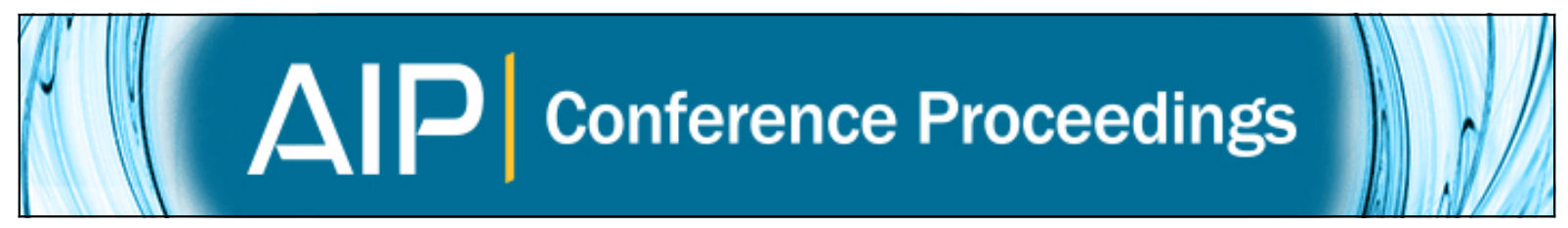

\title{
Improved Contact Algorithms for Implicit FE Simulation of Sheet Forming
}

S. Zhuang, M. G. Lee, Y. T. Keum, and R. H. Wagoner

Citation: AIP Conference Proceedings 908, 239 (2007); doi: 10.1063/1.2740818

View online: $\mathrm{http}: / / \mathrm{dx}$.doi.org/10.1063/1.2740818

View Table of Contents: http://scitation.aip.org/content/aip/proceeding/aipcp/908?ver=pdfcov

Published by the AIP Publishing

\section{Articles you may be interested in}

Decoupled Simulation Method For Incremental Sheet Metal Forming

AIP Conf. Proc. 908, 1501 (2007); 10.1063/1.2741021

Fully Integrated EAS-Based Solid-Shell Finite Elements in Implicit Sheet Metal Forming Simulations AIP Conf. Proc. 778, 751 (2005); 10.1063/1.2011312

Model Identification and FE Simulations: Effect of Different Yield Loci and Hardening Laws in Sheet Forming AIP Conf. Proc. 778, 371 (2005); 10.1063/1.2011248

Springback Simulation and Tool Surface Compensation Algorithm for Sheet Metal Forming AIP Conf. Proc. 778, 334 (2005); 10.1063/1.2011242

FE-Analysis of the Sheet Metal Forming Processes using Continuous Contact Treatment AIP Conf. Proc. 778, 191 (2005); 10.1063/1.2011216 


\title{
Improved Contact Algorithms for Implicit FE Simulation of Sheet Forming
}

\author{
S. Zhuang ${ }^{*}$, M. G. Lee ${ }^{*}$ Y. T. Keum ${ }^{\dagger}$, R. H. Wagoner ${ }^{*}$ \\ * Department of Materials Science and Engineering, The Ohio State University, 477 Watts Hall, 2041 College \\ Road, Columbus, OH, 43210, USA \\ ${ }^{\dagger}$ Division of Mechanical Engineering, Hanyang University, 17 Haengdang-Tong, Sungdong-Ku, Seoul 133-791 \\ South Korea
}

\begin{abstract}
Implicit finite element simulations of sheet forming processes do not always converge, particularly for complex tool geometries and rapidly changing contact. The SHEET-3 program exhibits remarkable stability and strong convergence by use of its special N-CFS algorithm and a sheet normal defined by the mesh, but these features alone do not always guarantee convergence and accuracy. An improved contact capability within the N-CFS algorithm is formulated taking into account sheet thickness within the framework of shell elements. Two imaginary surfaces offset from the mid-plane of shell elements are implemented along the mesh normal direction. An efficient contact searching algorithm based on the mesh-patch tool description is formulated along the mesh normal direction. The contact search includes a general global searching procedure and a new local searching procedure enforcing the contact condition along the mesh normal direction. The processes of unconstrained cylindrical bending and drawing through a drawbead are simulated to verify the accuracy and convergence of the improved contact algorithm.
\end{abstract}

Keywords: Sheet Forming; Implicit FM Method; N-CFS; Mesh Normal; Contact Distance Error; Contact Searching.

\section{INTRODUCTION}

In recent decades, sheet forming simulation has been a significant research field. Because of complex tool geometries, large relative movements and rapidly changing contact state, sheet forming simulation remains as an extremely challenging obstacle in industrial applications. Many finite element codes have been developed for sheet forming simulation. These codes are mainly classified as dynamic explicit (DE) or static implicit (SI). The DE codes can easily handle rapidly varying and highly nonlinear contact conditions and usually are not subject to the divergence problem, but they can be conditionally stable and are unsuitable for springback simulations [1]. Though the SI codes may have convergence problems, they are unconditionally stable and offer more reliability for springback simulations [2].

To robustly and stably simulate sheet forming, the choice of a reliable contact algorithm is critical. The contact algorithms are categorized as Lagrange multiplier, penalty-function, direct node projection, or augmented Lagrangian algorithms. The Lagrange multiplier algorithms introduce Lagrange multipliers, which add to the dimension of the global stiff matrix and may result in rigid body modes[3]. The accuracy of the penalty-function algorithms is severely dependent on the penalty parameter [4], and an unqualified penalty parameter usually results in illconditioning [3]. The direct node projection algorithms are often numerically unstable [5], especially for incompressible or nearly-incompressible problems [6]. The augmented Lagrangian algorithms can provide significant advantages over the Lagrange multiplier and the penalty algorithms [7] and lead to good conditioning [8].

The choice of tool description scheme and corresponding contact searching algorithm is also crucial for convergence and accuracy. Tool surfaces are usually described by analytical functions, triangular jewels, parametric patches, or typical mesh patches. Analytical-function description uses well defined mathematical functions. It can describe simple tools but has difficulty describing complicated tools. The triangular-jewel description depicts tool surfaces with triangular jewels based on equal-spaced control points. It has an advantage in that the contact searching along $Z$-axis direction is almost negligible. The fatal drawback of triangular-jewel description is the requirement of bulky memory for storing points, 
especially for tool surfaces with vertical parts. Parametric-patch description has difficulties in describing complex tools with many untrimmed and small surfaces [9] and requires complex contact searching and long computation time[10]. Mesh-patch description, by contrast, can easily and adequately describe arbitrarily complex tool surfaces with simple linear FE meshes and can provide simple geometry which has been used to organize an efficient contact search algorithm $[9,10]$.

As a special kind of augmented Lagrangian algorithm, N-CFS can overcome instability and divergence in deep drawing simulation [11, 12]. The mesh normal of each node is derived from neighboring elements [13] and the derivatives of tool surfaces are not required, so N-CFS is especially advantageous for the tool surfaces described by piecewise patches. However, the contact distance error defined in N-CFS neglects sheet thickness for shell elements. Moreover, previous implementations in SHEET-3 incorporated only analytical-function and triangular-jewel tool descriptions limiting the application to complex parts.

In this paper, an improvement of the N-CFS algorithm incorporating finite sheet thickness for shell elements is introduced, and an advanced contact searching algorithm based on mesh-patch tools is presented. Mesh-patch tool descriptions are incorporated and the mesh normal formulation is retained. For verification, the processes of unconstrained cylindrical bending and drawing through a drawbead are simulated and discussed.

\section{ACCOUNTING FOR SHELL THICKNESS}

The basic equilibrium equation is expressed as

$$
\mathbf{F}_{\mathrm{I}}(\Delta \mathbf{u})-\mathbf{F}_{\mathrm{E}}(\Delta \mathbf{u}, P)=\mathbf{0}
$$

where $\mathbf{F}_{\mathrm{I}}$ and $\mathbf{F}_{\mathrm{E}}$ are the internal and external forces, $\Delta \mathbf{u}$ is the incremental virtual displacement, and $P$ is the contact pressure. $\mathbf{F}_{\mathrm{I}}$ is calculated by the incremental internal work $\Delta \mathrm{W}_{\mathrm{I}}$ and expressed by

$$
\mathbf{F}_{\mathrm{I}}=\int_{V} \frac{\partial \Delta W_{\mathrm{I}}}{\Delta \mathbf{u}} \mathrm{dV}=\int_{V} \boldsymbol{\sigma}: \frac{\partial \Delta \boldsymbol{\varepsilon}}{\partial \Delta \mathbf{u}} \mathrm{dV}
$$

where $\sigma$ and $\Delta \varepsilon$ are Cauchy stress tensor and incremental strain tensor, respectively. By virtue of a modified Coulomb friction law with a smoothing factor $\phi[5,14], \mathbf{F}_{\mathrm{E}}$ is expressed by

$$
\mathbf{F}_{\mathrm{E}}=P(\mathbf{n}+\mu \phi \mathbf{t})
$$

where $\mathbf{n}$ and $\mathbf{t}$ are the mesh normal and tangential unit vectors, and $\mu$ is the friction coefficient.

The geometrically impenetrable constraint is set based on the contact distance error $\mathrm{D}_{\mathrm{N}}$ along the mesh normal direction between the sheet and tools, as

$$
\mathrm{D}_{\mathrm{N}}(\Delta \mathbf{u})=0
$$

The full set of governing equations, including the equilibrium equation $\mathrm{Eq}$. (1) and the impenetrable constraint Eq. (4), are expressed as follows:

$$
\left[\begin{array}{c}
\mathbf{F}_{\mathrm{I}}(\Delta \mathbf{u})-\mathbf{F}_{\mathrm{E}}(\Delta \mathbf{u}, P) \\
\mathrm{D}_{\mathrm{N}}(\Delta \mathbf{u})
\end{array}\right]=\mathbf{0}
$$

which is iteratively solved using a Newton-Raphson procedure and a Taylor series expansion to give

$$
\left[\begin{array}{cc}
\frac{\partial \mathbf{F}_{\mathrm{I}}}{\partial \Delta \mathbf{u}}-\frac{\partial \mathbf{F}_{\mathrm{E}}}{\partial \Delta \mathbf{u}} & -\frac{\partial \mathbf{F}_{\mathrm{E}}}{\partial P} \\
\frac{\partial \mathrm{D}_{\mathrm{N}}}{\partial \Delta \mathbf{u}} & 0
\end{array}\right]\left[\begin{array}{c}
\delta \Delta \mathbf{u} \\
\delta P
\end{array}\right]=\left[\begin{array}{c}
\mathbf{F}_{\mathrm{E}}-\mathbf{F}_{\mathrm{I}} \\
-\mathrm{D}_{\mathrm{N}}
\end{array}\right]
$$

In the N-CFS algorithm [11], the contact distance error $\mathrm{D}_{\mathbb{N}}$ is introduced as follows:

$$
\mathrm{D}_{\mathrm{N}}=\alpha\left|\mathbf{x}_{\text {tool }}-\mathbf{x}_{\text {rode }}\right|
$$

where $\alpha$ is either +1 or -1 , so that $\mathrm{D}_{\mathrm{N}}>0$ represents a non-penetrating node, and $\mathrm{D}_{\mathrm{N}}<0$ a penetrating node. Because the nodes for shell elements are defined at the sheet mid-plane, Eq. (7) simulates the mid-plane contact tools and ignores the sheet thickness.

To consider sheet thickness for shell elements, the improved N-CFS defines a new contact error $D_{N}$ at a punch point $\mathbf{x}_{\mathrm{p}}$ or a die point $\mathbf{x}_{\mathrm{d}}$ as

$$
\mathrm{D}_{\mathrm{N}}=\alpha\left|\mathbf{x}_{\mathrm{p}}-\mathbf{x}^{\mathrm{p}}\right|, \mathrm{D}_{\mathrm{N}}=\alpha\left|\mathbf{x}_{\mathrm{d}}-\mathbf{x}^{\mathrm{d}}\right|
$$

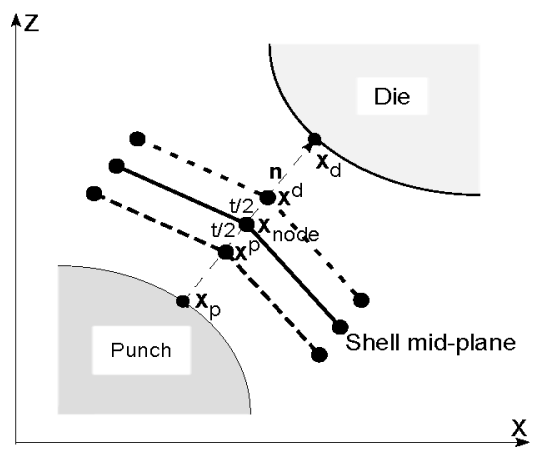

FIGURE 1. Top and bottom imaginary surfaces obtained by offsetting sheet mid-plane along mesh normal direction. 
Note that $\mathrm{D}_{\mathrm{N}}$ is still defined on the node $\mathbf{x}$. And $\mathbf{x}^{\mathrm{d}}$ and $\mathbf{x}^{\mathrm{p}}$ are the coordinates of the points on the top and bottom imaginary contact surfaces, generated by offsetting the mid-plane of the sheet along the mesh normal direction one-half of the sheet thickness, $t$, as illustrated in Fig.1. They are calculated by

$$
\mathbf{x}^{\mathrm{d}}=\mathbf{x}_{\text {node }}+t \mathbf{n} / 2, \mathbf{x}^{\mathrm{p}}=\mathbf{x}_{\text {node }}-t \mathbf{n} / 2
$$

where $t$ employs the updated sheet thickness, $t_{u}$.

The improved N-CFS differs not only by the different contact error, but also by the derivatives of external force and contact error, $\partial \mathbf{F}_{\mathrm{E}} / \partial \Delta P$ and $\partial \mathrm{D}_{\mathrm{N}} / \partial \Delta \mathbf{u}$ in Eq. (6). These derivatives are directly expressed with the first and second derivatives of sheet mesh surface $Z_{x}, Z_{y}, Z_{x x}, Z_{x y}$, and $Z_{y y}[11] . \quad Z_{x}$ and $Z_{x x}$ are examined at the punch contact points of a plane strain problem, as shown in Fig.2. When the sheet thickness is considered and the bottom surface of the sheet is assumed to contact the punch, $Z_{x}$ and $Z_{x x}$ are different from those neglecting sheet thickness.

\section{ADVANCED CONTACT SEARCHING}

Use of a non-uniform mesh to describe tools has many advantages, but there is a penalty in contact searching as compared with a uniform mesh. A contact searching algorithm was formulated consisting of general global searching and local searching. Global searching is to quickly but approximately find a set of candidate mesh patches. Local searching is to find the unique target segment from the set of candidate mesh patches obtained by global searching.

Consider an arbitrary node $i(\mathbf{x})$ with a line $l$ along a direction $\mathbf{n}$ and an arbitrary triangular segment $j$ with an outer normal $\mathbf{m}$, as illustrated in Fig.3(a).

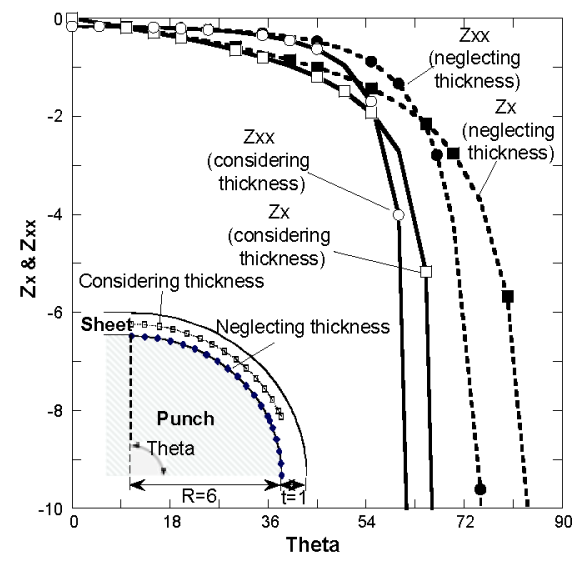

FIGURE 2. The first and second derivatives of sheet mesh surface $Z_{x}$ and $Z_{x x}$ at contact points, when considering thickness and neglecting thickness.
If the dot product $r(=\mathbf{n} \cdot \mathbf{m})$ is equal to zero, $l$ and $j$ are parallel, and $j$ is not the target segment. Otherwise, the intersection of the segment $j$ and the line $l, k(\mathbf{x})$, will be calculated by

$$
\begin{gathered}
\mathbf{m} \cdot\left(\mathbf{x}-\mathbf{x}_{\mathrm{A}}\right)=0 \\
\mathbf{x}=\mathbf{x}_{0}+\not \mathbf{n}
\end{gathered}
$$

which can subsequently give

$$
\mathbf{t}=\frac{\mathbf{m} \cdot\left(\mathbf{x}_{0}-\mathbf{x}_{\mathrm{A}}\right)}{\mathbf{n} \cdot \mathbf{m}}, \mathbf{x}=\mathbf{x}_{0}+\frac{\mathbf{m} \cdot\left(\mathbf{x}_{\mathrm{A}}-\mathbf{x}_{0}\right)}{\mathbf{n} \cdot \mathbf{m}} \mathbf{n}
$$

where $\mathbf{x}$ is the coordinate of the intersection point $k$.

The vector $\mathbf{V}_{\mathrm{Ak}}$ is decomposed with two bases $\mathbf{V}_{\mathrm{AB}}$ and $\mathbf{V}_{\mathrm{BC}}$, as shown in Fig.3(b) as

$$
\mathbf{V}_{\mathrm{Ak}}=p \mathbf{V}_{\mathrm{AB}}+q \mathbf{V}_{\mathrm{BC}}
$$

which can also be written in a matrix form as

$$
\left[\begin{array}{l}
x-a_{1} \\
y-a_{2} \\
z-a_{3}
\end{array}\right]=\left[\begin{array}{ll}
b_{1}-a_{1} & c_{1}-b_{1} \\
b_{2}-a_{2} & c_{2}-b_{2} \\
b_{3}-a_{3} & c_{3}-b_{3}
\end{array}\right]\left[\begin{array}{l}
p \\
q
\end{array}\right]
$$

Eq. (13) is solved by the following procedures:

(1) if $\left(b_{1}-a_{1}\right)\left(c_{2}-b_{2}\right)-\left(b_{2}-a_{2}\right)\left(c_{1}-b_{1}\right) \neq 0$,

$$
\left[\begin{array}{l}
p \\
q
\end{array}\right]=\left[\begin{array}{ll}
b_{1}-a_{1} & c_{1}-b_{1} \\
b_{2}-a_{2} & c_{2}-b_{2}
\end{array}\right]^{-1}\left[\begin{array}{l}
x-a_{1} \\
y-a_{2}
\end{array}\right]
$$

(2) if $\left(b_{2}-a_{2}\right)\left(c_{3}-b_{3}\right)-\left(b_{3}-a_{3}\right)\left(c_{2}-b_{2}\right) \neq 0$,

$$
\left[\begin{array}{l}
p \\
q
\end{array}\right]=\left[\begin{array}{ll}
b_{2}-a_{2} & c_{2}-b_{2} \\
b_{3}-a_{3} & c_{3}-b_{3}
\end{array}\right]^{-1}\left[\begin{array}{c}
y-a_{2} \\
z-a_{3}
\end{array}\right]
$$

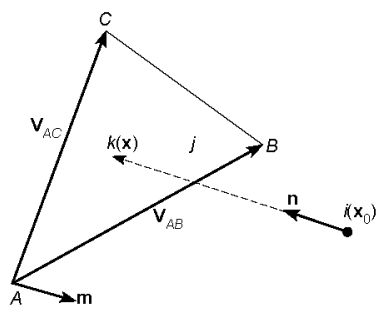

(a)

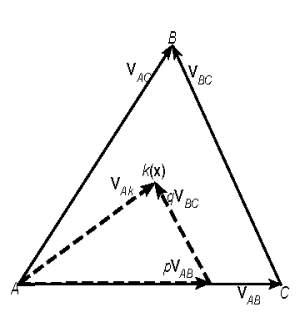

(b)
FIGURE 3. Local searching: (a) intersection between node $i$ and segment $j,(\mathrm{~b})$ the position of $k$ relative to segment $j$. 
(3) if $\left(b_{3}-a_{3}\right)\left(c_{1}-b_{1}\right)-\left(b_{1}-a_{1}\right)\left(c_{3}-b_{3}\right) \neq 0$,

$$
\left[\begin{array}{l}
p \\
q
\end{array}\right]=\left[\begin{array}{ll}
b_{3}-a_{3} & c_{3}-b_{3} \\
b_{1}-a_{1} & c_{1}-b_{1}
\end{array}\right]^{-1}\left[\begin{array}{l}
z-a_{3} \\
x-a_{1}
\end{array}\right]
$$

If $0 \leq \mathrm{q} \leq \mathrm{p} \leq 1$, point $k$ is located inside the segment $j$ and $j$ is the target segment.

\section{NUMERICAL EXAMPLES}

In the following examples, aluminum alloy 6111T4 sheet material is applied assuming the following material properties: Young's Modulus, $E=70.5 \mathrm{GPa}$; Poisson's ratio, $v=0.342$; Plastic anisotropy parameters, $r_{0}=0.894, r_{45}=0.611, r_{90}=0.660$; Hardening curve, $\bar{\sigma}=550.4(\bar{\varepsilon}+0.0093)^{0.223} \mathrm{MPa}$. The rolling direction (RD) of the sheet is longitudinal. Hill's 48 anisotropic yield function[15] is adopted.

\section{Unconstrained Cylindrical Bending}

Unconstrained cylindrical bending, a benchmark test problem in NUMISHEET2002 [16] which exhibits rapid contact change, is chosen to evaluate the accuracy and robustness of the developed contact algorithms. The schematic of tools is shown in Fig.4. NUMISHEET2002 provided a friction coefficient of 0.1348 . The experimental data of participant BE-01 [16] are compared with the simulations.

For comparison, the cases with constant thickness $t=t_{0}$ and the cases with the offset tools obtained by offsetting tools along the tool normal direction onehalf of sheet thickness $t_{0}$ are simulated. The punch force with respect to the punch displacement is shown in Fig.5. The punch displacement is defined relative to a starting condition where the sheet just makes contact with the tools on both surfaces. All the predicted results agree well with the experiment at lower punch displacements. At larger punch displacements, the importance of the modification to incorporate sheet thickness in N-CFS contact algorithm is clear. The simulations neglecting thickness greatly underestimate

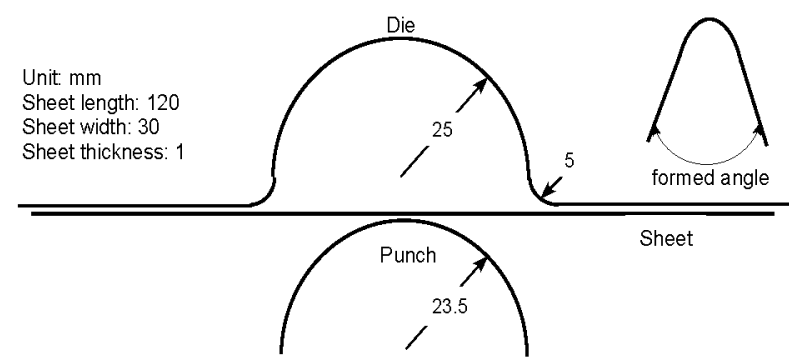

FIGURE 4. Schematic of unconstrained cylindrical bending the punch forces, as compared with experimental results, with a relative error up to $28 \%$ at the highest punch displacement. By contrast, the simulations considering thickness agree very well with the experiment, with a relative error of only $3 \%$. For the unconstrained cylindrical bending problem, there was no significant difference between using updated or constant sheet thickness and offset tools. Because the problem is bending-dominated, the thickness changes are very small. There is also no difference between an analytical-function tool description and the mesh-patch tool description.

The formed angles after forming are compared in Table 1. Note that the predicted angles of the simulations using zero thickness are larger than the experimental results, with a relative error up to $50 \%$. The predicted angles of the simulations considering thickness are similar to the experiment, with relative errors within $3 \%$. The simulations using updated thickness and constant thickness get the same results for this bending-dominated test. For both analyticalfunction description and mesh-patch description, the simulations using offset tools predict similar formed angles with those using updated thickness or constant thickness, and the relative errors are limited to $0.05 \%$. The mesh-patch and analytical-function descriptions yield similar formed angles for all the cases, with differences from $0.16 \%$ to $0.44 \%$.

The ability of the mesh-patch tool description to get results similar to those from the analytical-function description has been shown; now the efficiency of these two tool descriptions is compared. The total number of equilibrium iterations, which determines the total CPU for implicit methods, is compared. As

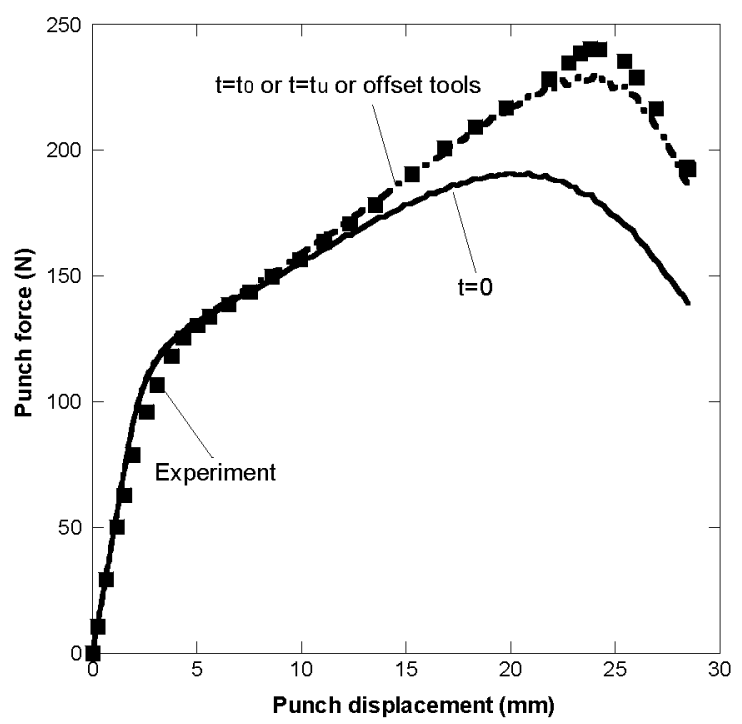

FIGURE 5. Punch force vs. punch displacement. Curves are indistinguishable for analytical-function tool description and mesh-patch tool description. 
TABLE 1. Influence of thickness on formed angles.

\begin{tabular}{|c|c|c|c|c|}
\hline Thickness & $t=0$ & $\mathrm{t}=\mathrm{t}_{\mathrm{u}}$ & $\mathrm{t}=\mathrm{t}_{\mathbf{0}}$ & Offset tools \\
\hline Analytical-function tool description & $31.50^{\circ}$ & $20.36^{0}$ & $20.36^{0}$ & $20.35^{0}$ \\
\hline Mesh-patch tool description & $31.55^{0}$ & $20.43^{0}$ & $20.43^{0}$ & $20.44^{0}$ \\
\hline Experiment & \multicolumn{4}{|c|}{$21.10^{0}$} \\
\hline
\end{tabular}

TABLE 2. Comparison of equilibrium iterations.

\begin{tabular}{lcccc}
\hline \multicolumn{1}{c}{ Thickness } & $\mathbf{t}=\mathbf{0}$ & $\mathbf{t}=\mathbf{t}_{\mathbf{u}}$ & $\mathbf{t}=\mathbf{t}_{\mathbf{0}}$ & Offset tools \\
\hline Analytical-function tool description & 1914 & 2447 & 2114 & 2269 \\
Mesh-patch tool description & 1991 & 1924 & 1995 & 2059 \\
\hline
\end{tabular}

shown in Table 2, the mesh-patch tool description needs $4 \%$ more equilibrium iterations than the analytical-function description for the simulations using zero thickness, but it needs fewer equilibrium iterations when considering sheet thickness, especially for the case using updated thickness, the analyticalfunction description uses $27 \%$ more equilibrium iterations than the mesh-patch tool description does. There is no consistent difference in the total number of iterations required by the two tool descriptions.

\section{Drawing through a Drawbead}

The schematic of drawing through a drawbead is shown in Fig.6. The width of the sheet, $2.0 \mathrm{~mm}$, is represented by two shell element of $1 \mathrm{~mm}$ width and is held constant (plane strain). Only the mesh-patch tool description is applied. First, in the clamping process, the male drawbead moves to a fixed location, with a corresponding drawbead interference defined relative to an initial state where the sheet just makes contact on both surfaces with the tools, and clamps the sheet into the female drawbead, using free boundary condition on the left edge and fixed boundary condition on the right edge of the sheet. Next, in the drawing process, the male and female drawbeads remain motionless and

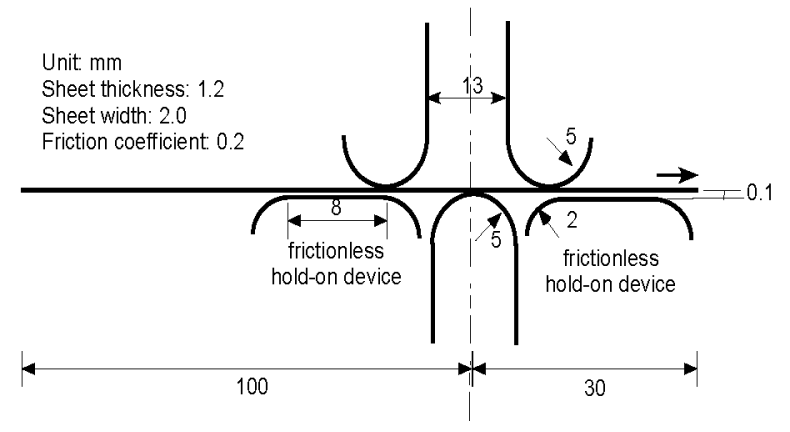

FIGURE 6. Schematic of drawing through a drawbead. the blank is pulled to the right at constant velocity of $1 \mathrm{~mm} / \mathrm{s}$ up to a drawing displacement of $45 \mathrm{~mm}$.

The simulated results of both clamping force and drawing force reach steady states when the draw distance exceeds $30 \mathrm{~mm}$. The averages and standard deviations of steady state clamping force and drawing force, at a drawbead interference of $12 \mathrm{~mm}$, are listed in the Table 3. The standard deviations are smaller than $1.3 \%$ of the corresponding average.

To study the influence of sheet thickness and sheet thinning on drawing process through a drawbead, five drawbead interferences are simulated. The steady state clamping forces and drawing forces are plotted in Fig.7. At lower drawbead interference, because the adjustment of tools for neglecting thickness increases the punch position corresponding to the die and counteracts the influence of sheet thickness, the simulation using zero thickness predicts larger drawing forces than the simulation using updated thickness. With the increase of the drawbead interference, the importance of thickness becomes more significant. The simulation using zero thickness, at a drawbead interference of $12 \mathrm{~mm}$, produces clamping forces up to $10 \%$ lower than those for finite updated thickness, and drawing forces up to $4 \%$ lower. The differences between finite updated sheet thickness and constant sheet thickness are about $1 \%$ for clamping force and about $2 \%$ for drawing force.

TABLE 3. Clamping forces and drawing forces at steady state at a drawbead interference of $12 \mathrm{~mm}$ for a $2 \mathrm{~mm}$ width blank.

\begin{tabular}{lcc}
\hline Thickness & $\begin{array}{c}\text { Clamping force } \\
(\mathbf{N})\end{array}$ & $\begin{array}{c}\text { Drawing force } \\
(\mathbf{N})\end{array}$ \\
\hline $\mathrm{t}=0$ & $373.6 \pm 4.9$ & $487.8 \pm 4.3$ \\
$\mathrm{t}=\mathrm{t}_{\mathrm{u}}$ & $414.5 \pm 5.2$ & $507.8 \pm 5.9$ \\
$\mathrm{t}=\mathrm{t} 0$ & $419.5 \pm 2.9$ & $516.1 \pm 3.7$ \\
\hline
\end{tabular}




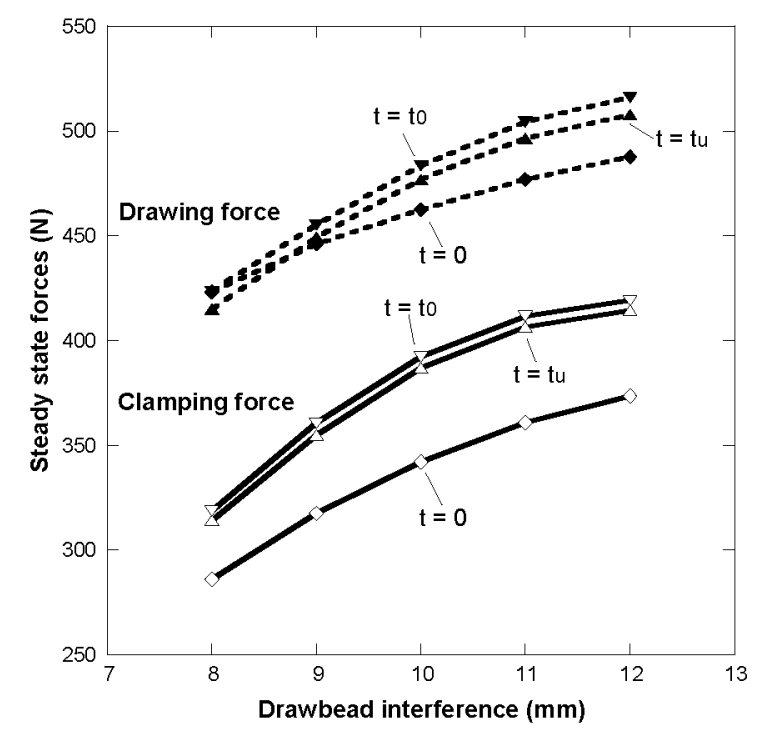

FIGURE 7. Clamping forces and drawing forces at steady states for various drawbead interferences.

\section{CONCLUSIONS}

An improved contact algorithm has been formulated, implemented and tested. It incorporates finite sheet thickness for contact with shell elements and makes use of mesh-patch tools with a two-stage contact search. Simulation results for two typical forming operations with and without the improvements were compared with experiments, with the following conclusions reached:

(1) Neglecting sheet thickness in cylinder bending produced errors of up to $28 \%$ in punch force and $50 \%$ in final formed angle. These were reduced to less than $3 \%$ when sheet thickness was accounted for.

(2) Neglecting sheet thickness in drawbead simulations produces clamping forces up to $10 \%$ lower than those for finite thickness, and drawing forces $4 \%$ lower. The differences in clamping forces and drawing forces between simulations with constant sheet thickness and updated sheet thickness are about $1 \%$ and $2 \%$, respectively.

(3) No significant differences were obtained between simulation results using analytical-function tools and mesh-patch tools.

(4) The new contact algorithm achieves better accuracy with no penalty in computational efficiency.

\section{ACKNOWLEDGEMENTS}

Support by NSF Grant (DMI-0355429) and the Ohio Supercomputer Center (PAS0080) is greatly appreciated. The authors would like to thank Dr. Thomas B. Stoughton (General Motors) and Dr. Subir Roy (Altair Engineering) for helpful discussions.

\section{REFERENCES}

1. N. He and R.H. Wagoner, "Springback Simulation in Sheet Metal Forming", NUMISHEET '96, The Ohio State University, Columbus, 1996, pp. 308-315.

2. L. Noels, L. Stainier, and J.P. Pontthot, "Combined implicit/explicit time-integration algorithms for the numerical simulation of sheet metal forming", Journal of Computational and Applied Mathematics, vol. 168 , 331-339 (2004).

3. P. Wriggers, "Computational contact mechanics", West Sussex, John Wiley \& Sons, 2002.

4. B. Nour-omid and P. Wriggers, "A two-level iteration method for solution of contact problems", Computer Methods in Applied Mechanics and Engineering, vol. 54, 131-144 (1986).

5. Y. Germain, K. Chung, and R.H. Wagoner, "A rigidvisco-plastic finite element program for sheet forming analysis", International Joumal of Mechanical Science, vol. 31, 1-24 (1989)

6. J.T. Oden, N. Kikuchi, and Y.J. Song, "Penalty-finite element methods for the analysis of stokesian flows", Computer Methods in Applied Mechanics and Engineering, vol. 31, 297-326 (1982).

7. J.C. Simo and T.A. Laursen, "An augmented Lagrangian treatment of contact problems involving friction", Computer \& Structures, vol. 42, 97-116 (1992).

8. K.J. Bathe, "Finite element procedures", New Jersey, Prentice-Hall, Inc., 1996.

9. A. Santos and A. Makinouchi, "Contact strategies to deal with different tool description in static explicit FEM for 3-D sheet metal forming simulation", Journal of Materials Processing Technology, vol. 50, 277-291 (1995).

10. D.Y. Yang, D.J. Yoo, I.S. Song, and J.H. Lee, "Investigation into tool surface description for finite element analysis of three-dimensional sheet metal forming process", Journal of Materials Processing Technology, vol. 45, 267-273 (1994).

11. D. Zhou and R.H. Wagoner, "An algorithm for improved convergence in sheet forming analysis", International Joumal of Mechanical Science, vol. 39 , 1363-1384 (1997).

12. M.J. Saran and R.H. Wagoner, "A Consistent Implicit Formulation for Nonlinear Finite Element Modeling with Contact and Friction, Part 1 - Theory", ASME Transactions of Journal of Applied Mechanics, vol. 58, 499-506 (1991).

13. Y.T. Keum, E. Nakamachi, R.H. Wagoner, and J.K. Lee, "Compatible description of tool surfaces and FEM meshes for analysing sheet forming operations", International Journal for Numerical Methods in Engineering, vol. 30, 1471-1502 (1990).

14. J.T. Oden and E.B. Pires, "Nonlocal and nonlinear friction laws and variational principles for contact problems in elasticity", Journal of Applied Mechanics, vol. 50(67), 67-76 (1983).

15. R. Hill, "Mathematical Theory of Plasticity", London, Oxford University Press, 1950.

16. "Proceeding of Numisheet 2002", NUMTSHEET 2002, Jeju Island, Korea, 2002. 